\title{
Clinical significance of concomitant extrahepatic autoimmune disease in patients with autoimmune liver disease
}

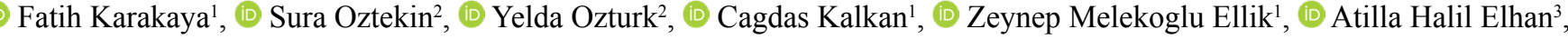 \\ D Irfan Soykan ${ }^{1}$, (D) Ramazan Idilman ${ }^{1}$ \\ ${ }^{1}$ Department of Gastroenterology, Ankara University School of Medicine, Ankara, Turkey; ${ }^{2}$ Department of Internal Medicine, Ankara University School \\ of Medicine, Ankara, Turkey; ${ }^{3}$ Department of Biostatistics, Ankara University School of Medicine, Ankara, Turkey
}

\begin{abstract}
Background and Aim: This study aimed to determine the presence of concomitant extrahepatic autoimmune disease (EAD) in patients with autoimmune liver disease (ALD) and the efficacy of the treatment response of ALD with the presence of any EAD.

Materials and Methods: Between January 2001 and November 2017, 241 patients with ALD were included in the study.

Results: Of the 241 patients, 88, 134, and 19 had autoimmune hepatitis $(\mathrm{AIH})$, primary biliary cholangitis (PBC), and overlap syndrome (OS), respectively. Thirty-one patients had cirrhosis: $77 \%$ and $23 \%$ had compensated and decompensated disease, respectively. The presence of at least one EAD was defined in $38.6 \%$ of the patients with ALD ( $n=93)$, and $12 \%$ of them had $\geq 1$ EAD. EAD was most commonly seen in patients with OS and PBC compared with those with AIH $(\mathrm{p}=0.036)$. Autoimmune thyroid disease was the most common association (20\%), followed by Sjogren syndrome $(12.0 \%)$. At the end of the follow-up period, 165 patients $(72 \%)$ had biochemical response. The presence of EAD did not affect the biochemical response.
\end{abstract}

Conclusion: EAD is most frequently seen in patients with ALD. The presence of EAD is not associated with the treatment response.

Keywords: Autoimmune liver disease; extrahepatic autoimmune disease.

\section{Introduction}

Autoimmune liver disease (ALD) consists of autoimmune hepatitis (AIH), primary biliary cholangitis (PBC), and primary sclerosing cholangitis (PSC) ${ }^{[1]}$ ALD may be associated with more than one subgroup as an overlap syndrome (OS). The term of OS is used to define the co-

How to cite this article: Karakaya F, Oztekin S, Ozturk Y, Kalkan C, Melekoglu Ellik Z, Elhan AH, et al. Clinical significance of concomitant extrahepatic autoimmune disease in patients with autoimmune liver disease. Hepatology Forum 2021; 2(1):3-6.

Received: October 26, 2020; Accepted: December 28, 2020; Available online: January 08,2021

Corresponding author: Fatih Karakaya; Ankara Universitesi Tip Fakultesi, Gastroenteroloji Anabilim Dali, Ankara, Turkey

Phone: +90 312363 6213; e-mail: mfkarakaya@yahoo.com

(c) (1) OPEN ACCESS

(1) This work is licensed under a Creative Commons Attribution-NonCommercial 4.0 International License.

๑ Copyright 2021 by Hepatology Forum - Available online at www.hepatologyforum.org existence of AIH with PBC or PSC. Autoimmune diseases are chronic, progressive conditions initiated by the loss of immunologic tolerance to self-antigens as a result of interactions of genetic, epigenetics, and environmental factors. ${ }^{[2-4]}$

Polyautoimmunity is defined as the presence of more than one autoimmune disease in a single patient. ${ }^{[1,2,4-8]}$ Approximately, one in three patients with ALD has a concomitant extrahepatic autoimmune disease (EAD), including endocrinologic, rheumatologic, dermatologic, or pulmonary diseases. ${ }^{[4-8]}$ This is mostly caused by the antigenic similarity in the hepatocyte or bile duct epithelial cell and the extrahepatic tissues, which activates innate and adoptive immune responses to similar antigenic structures. ${ }^{[1,2,4-8]}$ This study aimed to determine the presence of concomitant EAD in patients with ALD and the efficacy of the treatment response of primary disease with the presence of any EAD.

\section{Materials and Methods}

Patients: This is a single-center observational study. The medical data of patients diagnosed with ALD between January 2001 and November 2017 in the Liver Disease Outpatient Clinic, Department of Gastroenterology, Ankara University School of Medicine, were retrospectively evaluated. ALD was defined clinically, biochemically, and histologically when available. ICD-10 codes were used to identify disease and its complications. The patients were categorized as AIH, PBC, and OS using ICD-10 diagnostic codes. A diagnosis of ALD was established based on the international criteria, ${ }^{[9,10]}$ without any other cause of chronic liver disease. Patients with AIH were treated conventionally with corticosteroids and azathioprine. Prednisolone or prednisone was tapered to a maintenance dose of 5-7.5 mg/day. ${ }^{[9]}$ Patients with PBC were treated with ursodeoxycholic acid (UDCA) at a dose of $10-15 \mathrm{mg} /$ $\mathrm{kg} /$ day ${ }^{[10]}$ Complications of cirrhosis, including ascites, variceal bleeding, hepatic encephalopathy, hepatorenal syndrome, and hepatocellular carcinoma (HCC), were evaluated. Data were collected from outpatient visit charts. This study was approved by the local ethical committee of Ankara University School of Medicine.

Methods: Serum alanine aminotransferase, aspartate aminotransferase (AST), gamma glutamyl transpeptidase (GGT), alkaline phosphatase (ALP), bilirubin, and complete blood cell counts were measured by our central laboratory. Serological markers for viral infections (anti-HAV IgM, HBsAg, anti-HBs, HBeAg, anti-HBe, antiHBc IgG, anti-HCV, anti-HEV, anti-cytomegalovirus, anti-herpes simplex virus, and anti-Epstein-Barr virus), serum iron, ferritin, ceruloplasmin, and alpha-1 antitrypsin levels were measured, and serological studies for 


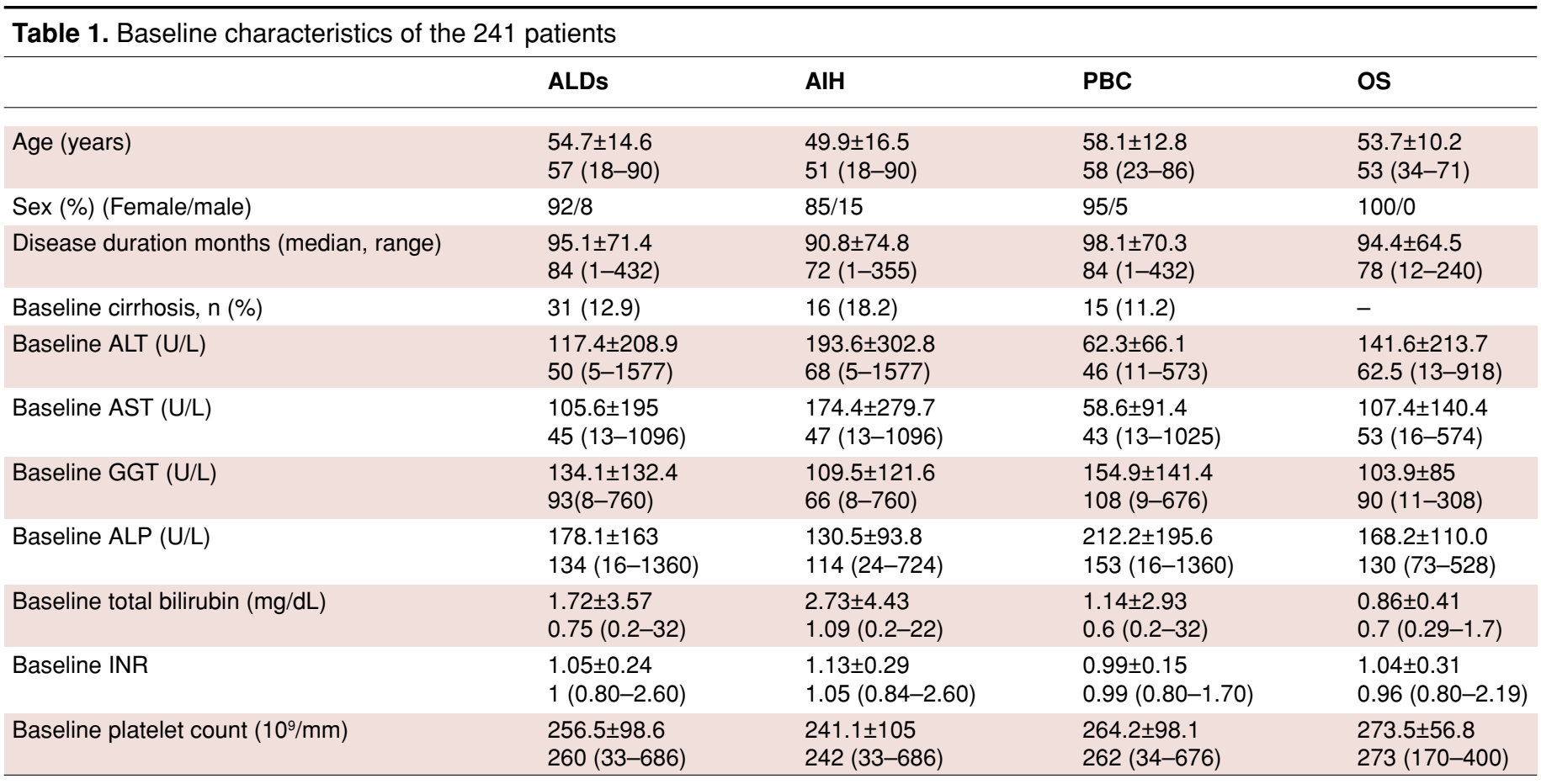

Data are presented as mean \pm SD, median (min, max). AlH: Autoimmune hepatitis; PBC: Primary biliary cholangitis; OS: Overlap syndrome; AST: Aspartate aminotransferase; GGT: Gamma glutamyl transpeptidase; ALP: Alkaline phosphatase.

anti-nuclear antibody, anti-smooth muscle antibody, anti-liver kidney membrane-1, and anti-mitochondrial antibodies were performed. All patients underwent abdominal sonography confirming the presence of cirrhosis, its complications, and HCC. Laboratory tests were performed at diagnosis and during the follow-up period.

Definitions: EAD was defined as patients with at least one or more of the following conditions including autoimmune thyroid disease (autoimmune thyroiditis, Hashimoto's disease, Graves' disease), Sjogren's syndrome, diabetes mellitus type 1, autoimmune skin disease (alopecia, vitiligo, psoriasis, dermatitis herpetiformis), systemic lupus erythematous, celiac disease, vasculitis, rheumatoid arthritis (RA), connective tissue disease, inflammatory bowel disease, polymyositis, uveitis, systemic sclerosis, PSC, Churg-Strauss syndrome, autoimmune thrombocytopenia and autoimmune hemolytic anemia. ${ }^{[5-8]}$

Biochemical response in patients with $\mathrm{AIH}$ was defined as a complete normalization of serum aminotransferases and immunoglobulin G levels. ${ }^{[9]}$ In patients with PBC, the response criteria are based on the improvement of serum ALP, AST, and bilirubin levels after 12 months of UDCA treatment based on Paris-II criteria (ALP $<1.5 \times$ upper limit of normal [ULN], AST $<1.5 \times$ ULN, normal bilirubin). ${ }^{[10]}$

Follow-up: Patients were seen at regular intervals in an outpatient clinic during the follow-up period. Cirrhosis and its complications were evaluated. Further investigations included surveillance for HCC with radiological imaging and alpha-fetoprotein determinations every 6 months. If necessary, dynamic computed tomography or magnetic resonance imaging was performed.

\section{Statistical Analysis}

Mean, standard deviation, median, minimum, maximum, frequency, and percent were used for descriptive statistics. Categorical variables were assessed using the chi-squared test. Comparison between two groups was assessed using the Mann-Whitney U test for non-normally distributed variables. A $p<0.05$ was considered significant.

\section{Results}

This study included 241 patients (female/male: $92 \% / 8 \%$ ), with a median age of 57.0 years (range: 18-90 years): 88 patients (median age: 51.0 years, range: $18-90$ years) with AIH, 134 patients (median age: 58.0 years, range: $23-86$ years) with $\mathrm{PBC}$, and 19 patients (median age: 53.0 years, range: $34-71$ years) with OS. Female sex was predominant (92\%). Thirty-one patients $(12.9 \%)$ had cirrhosis (16 AIH, $15 \mathrm{PBC})$. Among 31 patients with cirrhosis, 24 (77.4\%) and 7 (22.6\%) had compensated and decompensated disease. The median disease duration was 84 months (range: 1-432 months). Table 1 shows the patients' baseline characteristics.

The presence of at least one EAD was defined in $38.6 \%$ of the patients with ALDs $(n=93)$. EAD was most frequently seen in patients with OS (52.6\%) and PBC (43.3\%) compared with patients with AIH (28.1\%, $\mathrm{p}=0.036)$. Autoimmune thyroid disease was the most common association $(20.3 \%, 49 / 241)$, followed by Sjogren syndrome in 29 patients $(12 \%)$, RA in $8(3.3 \%)$, celiac disease in 7 (2.9\%), diabetes mellitus type 1 in 6 $(2.5 \%)$, autoimmune skin disease in $5(2.1 \%)$, and miscellaneous (inflammatory bowel disease, uveitis, systemic lupus erythematosus, systemic sclerosis, PSC, Churg-Strauss syndrome, and autoimmune thrombocytopenia) in 28 patients (11.6\%) (Table 2). Concomitant autoimmune thyroid disease was more commonly observed in patients with OS compared with patients with $\mathrm{AIH}(36.8 \%$ vs $13.6 \%$, p $=0.04)$, whereas Sjogren syndrome was slightly more common in patients with $\mathrm{PBC}(16.4 \%$ vs $6.8 \%$ in $\mathrm{AIH}$ and $5.3 \%$ in OS, respectively, $\mathrm{p}=0.06)$. In addition, 29 patients $(12 \%)$ had $\geq 1 \mathrm{EAD}$. The frequency was not significantly different among the groups (13.6\% in AIH, $11.2 \%$ in PBC, and $10.5 \%$ in OS) ( $p=0.6)$. 
Table 2. Frequency of EAD in patients with ALD

\begin{tabular}{|c|c|c|c|c|c|c|c|c|}
\hline & \multicolumn{2}{|c|}{ ALDs $(n=241)$} & \multicolumn{2}{|c|}{ AlH $(n=88)$} & \multicolumn{2}{|c|}{ PBC $(n=134)$} & \multicolumn{2}{|c|}{ OS $(n=19)$} \\
\hline Sjogren syndrome & 29 & 12 & 6 & 6.8 & 22 & 16.4 & 1 & 5.3 \\
\hline Rheumatoid arthritis & 8 & 3.3 & 3 & 3.4 & 5 & 3.7 & 0 & 0 \\
\hline Skin disease & 5 & 2.1 & 4 & 4.5 & 0 & 0 & 1 & 5.3 \\
\hline Miscellaneous & 23 & 9.5 & 10 & 11.4 & 11 & 8.2 & 2 & 10.5 \\
\hline
\end{tabular}

EAD: Extrahepatic autoimmune disease; ALD: Autoimmune liver disease; AlH: Autoimmune hepatitis; PBC: Primary biliary cholangitis; OS: Overlap syndrome.

Table 3. Biochemical response rate with univariate analysis

\begin{tabular}{llll}
\hline & Biochemical response & Biochemical non-response & p \\
\hline Age (years) & 57 & 57 & 0.572 \\
\hline Gender (\%) (Female/male) & $71 / 82$ & $29 / 18$ & 0.313 \\
Baseline ALT (U/L) & 49 & 52.5 & 0.691 \\
Baseline AST (U/L) & 43 & 52 & $0.03^{*}$ \\
Baseline GGT (U/L) & 82.5 & 138.5 & $0.024^{*}$ \\
Baseline ALP (U/L) & 123.5 & 160 & \\
Baseline total bilirubin (mg/dL) & 0.7 & 0.7 & \\
Baseline platelet count (10\%/mm) & 263 & 239 & \\
Cirrhosis (\%) & 60 & 40 & \\
No cirrhosis (\%) & 73.5 & 26.5 & \\
Presence of EAD (\%) & 78.3 & 21.7 & \\
Without EAD (\%) & 67.4 & 32.6 & 0.394 \\
AlH (\%)/PBC (\%)/OS (\%) & $76.5 / 69.2 / 68.4$ & $23.5 / 30.8 / 31.6$ \\
\hline *: Significant p-values as $<0.05$. Data are presented as median. EAD: Extrahepatic autoimmune disease; ALD: Autoimmune liver disease; AlH: Autoimmune hepatitis; \\
PBC: Primary biliary cholangitis; OS: Overlap syndrome; AST: Aspartate aminotransferase; GGT: Gamma glutamyl transpeptidase; ALP: Alkaline phosphatase. \\
\hline
\end{tabular}

At the end of the follow-up period, 165 patients had biochemical response, 65 had non-response, and 11 patients (7 AIH, 4 PBC) died. The biochemical response rates in patients with $\mathrm{AIH}, \mathrm{PBC}$, and $\mathrm{OS}$ were $76.5 \%, 69.2 \%$, and $68.4 \%$, respectively. Of the remaining 230 patients (21.7\%), 50 had cirrhosis (18 AIH, 28 PBC, and 4 OS). Among them, $68 \%(\mathrm{n}=34)$ and $32 \%(\mathrm{n}=16)$ had compensated (10 AIH, $21 \mathrm{PBC}$ and 3 OS) and decompensated disease (8 AIH, 7 PBC, and 1 OS). At end of the follow-up period, cirrhosis developed in 30 patients without cirrhosis at baseline ( $8 \mathrm{AIH}, 18 \mathrm{PSC}$, and 4 OS). Of the 30 patients, decompensated cirrhosis developed in 7 (23.3\%, $3 \mathrm{AIH}, 3 \mathrm{PSC}$, and $1 \mathrm{OS})$.

Among baseline patients' characteristics include age, gender, liver injury and cholestatic tests, type of ALD, the presence of cirrhosis, the presence of EAD, with univariate analysis, baseline low AST, GGT, and ALP levels were associated with biochemical response (Table 3). However, this was not statistically significant in multivariable analysis.

\section{Discussion}

The present study investigated the frequency, type, and clinical significance of concomitant EAD in patients with ALD in a single tertiary center. The presence of at least one EAD was found in $39 \%$ of the pa- tients with ALD, and $\geq 1$ EAD was observed in $12 \%$ of the patients. Female sex was predominant (96\%). EAD was most commonly seen in patients with OS and PBC compared with those with AIH $(\mathrm{p}=0.036)$. Among the EAD, autoimmune thyroid disease was most commonly encountered (20\%), followed by Sjogren syndrome (12\%). This finding is compatible with previous studies, ${ }^{[5,7,8,11-13]}$ which reported a close association between EAD and ALD, and autoimmune thyroid disease was most frequently encountered. Because the association between $\mathrm{AIH}$ and $\mathrm{EAD}$ is well known, these results indicate that high frequency of concomitant EAD was also seen in patients with OS and PBC.

The prevalence and pattern of concomitant EAD in patients with ALD vary due to different geographical areas, genetic background, and study populations. Floreani et al. ${ }^{[5]}$ reported a high prevalence of EAD $(61 \%)$ in Italian patients with PBC. Several investigators previously reported the prevalence of EAD in patients with ALD between $28.3 \%$ and $43.6 \%{ }^{[8,11,13]}$ In the present study, the overall prevalence of EAD in patients with ALD was $38.6 \%$, whereas it was $43.3 \%$ in patients with PBC. These results were compatible with previous reports ${ }^{[8,11,13]}$ but lower than those of the Italian group. Autoimmune thyroid disease was the most frequently observed, with a prevalence of $10 \%$ and $15 \%,{ }^{[5,8,11-}$ ${ }^{13]}$ which was similar to our results $(20 \%)$. The differences mostly seen 
in the pattern of the EAD. Some centers reported a high prevalence of Sjogren syndrome with/without Raynaud phenomenon and autoimmune skin disease, ${ }^{[5,11]}$ the other centers did not. ${ }^{[8,13]}$ The prevalence of Sjogren syndrome, Raynaud phenomenon, and autoimmune skin disease was $34 \%, 18 \%$, and $5 \%$ in Italian patients with PBC, respectively. In the present study, the prevalence of Sjogren syndrome in patients with PBC was $16.4 \%$, which was lower than that of their Italian counterparts, whereas autoimmune skin disease was not observed in patients with PBC. Unfortunately, Raynaud phenomena was not evaluated. This discrepancy may be explained by the heterogenous study population and different geographical distributions. Of note, $2.2 \%$ frequency of celiac disease was observed in patients with $\mathrm{PBC}$, similar to the Italian study, which reported $1.4 \%{ }^{[5]}$

Several investigators reported that the presence of EAD was not associated with treatment response, did not affect disease outcome, and were not related with the onset of major events during the follow-up period. ${ }^{[5,11-14]}$ Teufel et al. ${ }^{[12]}$ reported that the presence of EAD in patients with AIH did not affect treatment response and relapse rate. In the present study, no statically significant differences among the patient groups in the treatment response based on biochemical response. The presence of EAD in patients with $\mathrm{AIH}, \mathrm{PBC}$, and OS did not affect biochemical response, disease progression, and patient survival. This finding confirms that of previous studies. ${ }^{[11-14]}$

In conclusion, EAD was most frequently seen in patients with ALD. The presence of EAD was not associated with treatment response and disease progression. Asymptomatic patients with ALD may be identified as a result of the presence the EAD.

Ethics Committee Approval: This study was approved by the local ethical committee of Ankara University School of Medicine (Approval date: 05.09.2016, approval number: 14-671-16).

Peer-review: Externally peer-reviewed.

Author Contributions: Concept - RI, IS, FK, CK; Design - RI, IS; Supervision - RI, IS, AHE; Fundings - RI, AHE; Materials - RI, FK, CK, ZME, SO, YO; Data Collection and/or Processing - FK, CK, ZME, SO, YO; Analysis and/or Interpretation - RI, AHE; Literature Search - RI, FK, ZME, SO, YO; Writing RI, FK; Critical Reviews - RI, IS.

Conflict of Interest: The authors have no conflict of interest to declare.

Financial Disclosure: The authors declared that this study has received no financial support.

\section{References}

1. Floreani A, De Martin S, Secchi MF, Cazzagon N. Extrahepatic autoimmunity in autoimmune liver disease. Eur J Intern Med 2019;59:1-7.

2. Johar A, Sarmiento-Monroy JC, Rojas-Villarraga A, Silva-Lara MF, Patel $\mathrm{HR}$, Mantilla RD, et al. Definition of mutations in polyautoimmunity. J Autoimmun 2016;72:65-72.

3. Carbone M, Neuberger JM. Autoimmune liver disease, autoimmunity and liver transplantation. J Hepatol 2014;60(1):210-223.

4. Floreani A, Franceschet I, Cazzagon N. Primary biliary cirrhosis: overlaps with other autoimmune disorders. Semin Liver Dis 2014;34(3):352-360.

5. Floreani A, Franceschet I, Cazzagon N, Spinazzè A, Buja A, Furlan P, et al. Extrahepatic autoimmune conditions associated with primary biliary cirrhosis. Clin Rev Allergy Immunol 2015;48(2-3):192-197.

6. Anaya JM. The diagnosis and clinical significance of polyautoimmunity. Autoimmun Rev 2014;13(4-5):423-426.

7. Wong GW, Heneghan MA. Association of Extrahepatic Manifestations with Autoimmune Hepatitis. Dig Dis 2015;33(Suppl 2):25-35.

8. Efe C, Wahlin S, Ozaslan E, Berlot AH, Purnak T, Muratori L, et al. Autoimmune hepatitis/primary biliary cirrhosis overlap syndrome and associated extrahepatic autoimmune diseases. Eur J Gastroenterol Hepatol 2012;24(5):531-534.

9. Czaja AJ. Diagnosis and Management of Autoimmune Hepatitis: Current Status and Future Directions. Gut Liver 2016;10(2):177-203.

10. Invernizzi P, Floreani A, Carbone M, Marzioni M, Craxi A, Muratori L, et al. Primary Biliary Cholangitis: advances in management and treatment of the disease. Dig Liver Dis 2017;49(8):841-846.

11. Muratori P, Fabbri A, Lalanne C, Lenzi M, Muratori L. Autoimmune liver disease and concomitant extrahepatic autoimmune disease. Eur J Gastroenterol Hepatol. 2015 Oct;27(10):1175-1179.

12. Teufel A, Weinmann A, Kahaly GJ, Centner C, Piendl A, Wörns M, et al. Concurrent autoimmune diseases in patients with autoimmune hepatitis. J Clin Gastroenterol 2010;44(3):208-213.

13. Efe C, Torgutalp M, Henriksson I, Alalkim F, Lytvyak E, Trivedi H, et al. Extrahepatic autoimmune diseases in primary biliary cholangitis: Prevalence and significance for clinical presentation and disease outcome. J Gastroenterol Hepatol 2020 Aug 13. doi: 10.1111/jgh.15214. [Epub ahead of print]

14. Gershwin ME, Selmi C, Worman HJ, Gold EB, Watnik M, Utts J, et al; USA PBC Epidemiology Group. Risk factors and comorbidities in primary biliary cirrhosis: a controlled interview-based study of 1032 patients. Hepatology 2005;42(5):1194-1202. 